\title{
Neuroinflammation PET Imaging: Current Opinion and Future Directions
}

\author{
Poorva Jain ${ }^{1}$, Aisling M. Chaney ${ }^{1}$, Mackenzie L. Carlson ${ }^{1,2}$, Isaac M. Jackson ${ }^{1}$, Anoushka Rao ${ }^{1}$, and Michelle L. James ${ }^{1,3}$ \\ ${ }^{1}$ Department of Radiology, Molecular Imaging Program at Stanford, Stanford University, Stanford, California; ${ }^{2}$ Department of \\ Bioengineering, Stanford University, Stanford, California; and ${ }^{3}$ Department of Neurology and Neurological Sciences, Stanford \\ University, Stanford, California
}

\begin{abstract}
Neuroinflammation is a key pathologic hallmark of numerous neurologic diseases, however, its exact role in vivo is yet to be fully understood. PET imaging enables investigation, quantification, and tracking of different neuroinflammation biomarkers in living subjects longitudinally. One such biomarker that has been imaged extensively using PET is translocator protein $18 \mathrm{kDa}$ (TSPO). Although imaging TSPO has yielded valuable clinical data linking neuroinflammation to various neurodegenerative diseases, considerable limitations of TSPO PET have prompted identification of other more cell-specific and functionally relevant biomarkers. This review analyzes the clinical potential of available and emerging PET biomarkers of innate and adaptive immune responses, with mention of exciting future directions for the field.
\end{abstract}

Key Words: biomarker validation; clinical imaging; molecular imaging; neuroinflammation; positron emission tomography

J Nucl Med 2020; 61:1107-1112

DOI: $10.2967 /$ jnumed.119.229443

\section{$\mathbf{N}$} and infiltration of peripheral leukocytes into the central nervous system (CNS). Microglia are the first line of defense and primary resident immune cells in the brain and spinal cord. These cells surveil their surrounding microenvironment to maintain homeostasis and protect against infection and damage (1). Upon acute activation, microglia initiate inflammatory signaling cascades through release of cytokines or chemokines and generation of reactive oxidative species to defend against pathogens and mitigate injury $(1,2)$. This activation is usually transient and resolves as basal physiology is restored. However, under aberrant conditions microglia may stay chronically active, turning a protective mechanism maladaptive (1).

Mounting data from rodent models of CNS diseases and genomewide association studies highlight the integral role of the immune system and its dysregulation in neurodegenerative diseases, including Alzheimer disease (AD), Parkinson disease (PD), and multiple sclerosis (MS) (2). In such diseases, microglia can take on both neurotoxic and neuroprotective roles and often lose the ability to function properly (e.g., ineffective and unregulated phagocytosis). Beyond microglia, several other cells are often involved in neurotoxic

Received Mar. 17, 2020; revision accepted May 20, 2020.

For correspondence or reprints contact: Michelle James, Department of Radiology, Stanford University, 1201 Welch Rd., P-206, Stanford, CA 94305. E-mail: mljames@stanford.edu

Published online Jul. 3, 2020.

COPYRIGHT (C 2020 by the Society of Nuclear Medicine and Molecular Imaging. actions, in the context of neuroinflammation, including astrocytes, endothelial cells, and oligodendrocytes, in addition to infiltrating peripheral innate immune cells (e.g., macrophages, monocytes, and neutrophils) and adaptive immune cells (e.g., B and T lymphocytes) (1-3).

Immune cell activation states are context-dependent (1) and therefore cannot be sufficiently studied through isolating or culturing cells, analyzing transcriptomic data, or using biopsy sampling alone. There is a need for noninvasive techniques that enable longitudinal visualization of neuroimmune responses in vivo. PET is widely used in both research and clinical settings for studying biologic processes in living subjects and is an indispensable tool for early detection or diagnosis of disease, real-time therapy monitoring, and patient stratification in clinical trials. Imaging the CNS using PET requires active transport or passive diffusion of a radiolabeled molecule across the blood-brain barrier (BBB). Thus, successful radiotracer design for imaging neuroinflammation typically employs the use of small molecules and requires striking a balance between achieving optimal binding characteristics (e.g., high affinity, high selectivity, and low nonspecific binding) and maintaining properties conducive to transport across the BBB (e.g., molecular weight of less than $500 \mathrm{Da}$, calculated $\log \mathrm{P}$ between 2 and 3, and total polar surface area between 20 and 120 $\AA^{2}$ ) (4). Apart from small molecules, biologics (e.g., monoclonal antibodies [mAbs] and minibodies) and other large molecules (e.g., adenoviruses and dendrimers) are increasingly being explored for CNS imaging. Given their larger sizes, these agents often require additional considerations or creative strategies to ensure adequate $\mathrm{BBB}$ penetration (e.g., transferrin conjugation, active transport, or the use of focused ultrasound to temporarily disrupt the $\mathrm{BBB}$ ).

Several PET radiotracers have been developed for candidate biomarkers of neuroinflammation, including translocator protein $18 \mathrm{kDa}$ (TSPO), cannabinoid receptor 2 (CB2R), and cyclooxygenase enzymes. However, many of these imaging targets face challenges that limit their overall success, such as suboptimal cellular specificity and low signal-to-noise ratios (SNRs). Accordingly, there is an evolving shift in mindset to conduct rigorous and systematic validation of candidate biomarkers before engaging in time- and resource-intensive radiotracer development. Criteria for such validations include an adequate concentration of available target binding sites (maximum specific binding $>10 \mathrm{nM}$ ), a sufficient change in target expression in relevant CNS diseases (correlating with disease severity), a low basal expression in the CNS (ensuring reasonable SNR), high specificity for a cell type of interest, and functional relevance. This review briefly describes state-of-the-art approaches to neuroinflammation PET imaging 
and highlights recent key advancements in the field while using the above criteria to critically analyze the potential clinical impact and foreseeable obstacles to widespread use of existing and emerging radiotracers.

\section{TSPO PET}

To date, the most extensively explored target for imaging neuroinflammation is TSPO $(1,5)$. This mitochondrial protein is involved in numerous physiologic processes, including neuronal survival and steroidogenesis (1). Although TSPO is densely distributed in many peripheral tissues under homeostatic conditions, basal CNS expression is low. During neuroinflammatory states, TSPO expression becomes significantly upregulated primarily in activated microglia, reactive astrocytes, and CNS-infiltrating myeloid-lineage cells (e.g., macrophages and monocytes) (1,5). Thus, TSPO can serve as an effective target for probing the temporal dynamics of immune activation across diseases.

Historically, ${ }^{11} \mathrm{C}-\mathrm{PK} 11195$ has been the most widely used TSPO radiotracer. Although PET studies using ${ }^{11} \mathrm{C}-\mathrm{PK} 11195$ have provided numerous valuable insights on glial or macrophage activation in different neurologic disorders, including dementia and stroke (1), its poor brain permeability and high plasma protein binding have limited its sensitivity and overall clinical utility. Thus, many second-generation TSPO tracers were developed to improve brain uptake and increase sensitivity for detecting subtle alterations in neuroinflammation. One of the most promising second-generation tracers is ${ }^{11} \mathrm{C}$-DPA-713, which displays favorable in vivo tracer characteristics (higher brain uptake) and superior sensitivity (e.g., improved SNR and specific binding) for detecting neuroinflammation in patients and rodent models of neurologic disease compared with ${ }^{11} \mathrm{C}$-PK11195 and other TSPO tracers (6-8). Notably, Yokokura et al. identified multiple brain regions containing elevated ${ }^{11} \mathrm{C}-\mathrm{DPA}-713$ binding in AD patients compared with healthy controls-a difference that was not detected with ${ }^{11}$ C-PK11195 (6). In addition, a negative correlation was identified between cognitive function and ${ }^{11} \mathrm{C}$-DPA-713 binding, further highlighting its sensitivity and potential clinical utility. Another promising second-generation tracer is ${ }^{11} \mathrm{C}-\mathrm{PBR} 28$, which has been applied clinically in a range of neurologic disorders, including dementia and chronic pain (5,9). Importantly, ${ }^{11} \mathrm{C}-\mathrm{PBR} 28$ has been used to monitor immunomodulatory therapies in PD patients, wherein decreased binding of ${ }^{11} \mathrm{C}-\mathrm{PBR} 28$ was found after treatment with myeloperoxidase inhibitor AZD3241 (Fig. 1). This provides proof-of-concept data that TSPO PET could be used for clinical therapy monitoring (10). Similar to ${ }^{11} \mathrm{C}$-DPA713, ${ }^{11} \mathrm{C}$-PBR28 exhibits greater affinity for TSPO and higher levels of specific binding than ${ }^{11} \mathrm{C}-\mathrm{PK} 11195(5,8)$. However, its binding potential is lower than that observed with ${ }^{11} \mathrm{C}$-DPA-713 in healthy humans (1.2 vs. 7.3) (8).

An important limitation regarding clinical application of second-generation tracers is their universal sensitivity to the rs6971 TSPO polymorphism, which results in high-, mixed-, and low-affinity binders (5). Although the frequency of low-affinity binders is much lower than that of high-affinity and mixed-affinity binders, the lack of quantifiable TSPO signal in low-affinity binders limits the number of patients eligible for second-generation TSPO PET and necessitates time-intensive genetic testing to validate patient eligibility before imaging. Recent advances in TSPO PET have focused on the development of third-generation tracers insensitive to the rs6971 polymorphism. ${ }^{11} \mathrm{C}$-ER176, a quinazoline

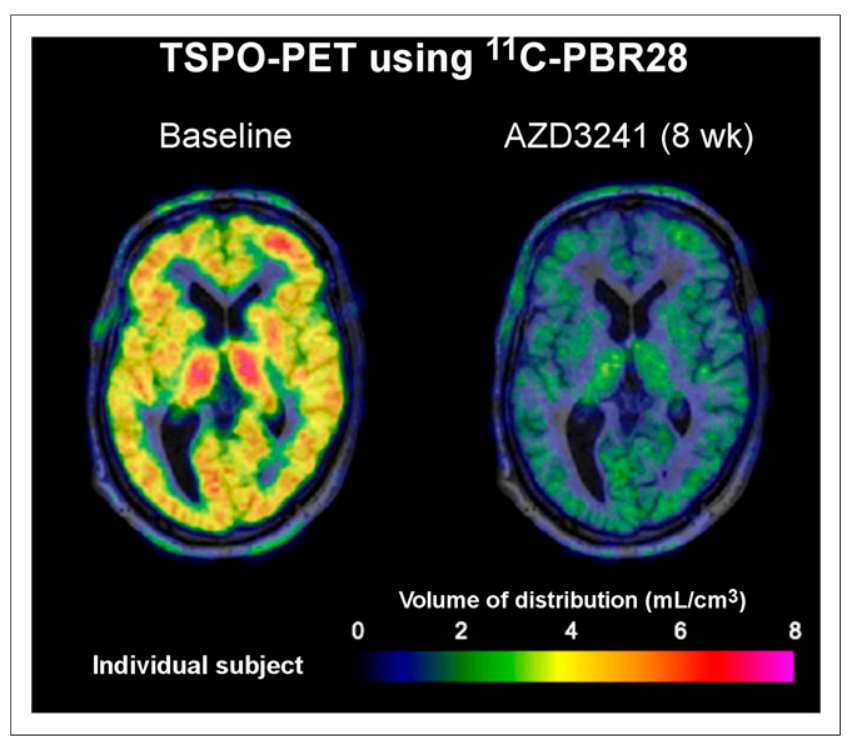

FIGURE 1. TSPO PET imaging shows response to immunomodulatory therapy, myeloperoxidase inhibitor AZD3241, in representative PD patient. (Modified from (10).)

analog of PK11195, was identified as a promising candidate with limited sensitivity to the rs6971 polymorphism in vitro, although in vivo investigation revealed lower brain uptake in low-affinity binders (vs. high-affinity and mixed-affinity binders) $(1,8)$. Nonetheless, ${ }^{11} \mathrm{C}$-ER176 exhibits sufficient binding potential (relative to non-displaceable tissue uptake) to permit imaging of low-affinity binders and appears to have enhanced specificity and SNR compared with previous tracers due to the absence of brain-penetrable radiometabolites.

Although TSPO PET remains the most widely used technique for clinical neuroinflammation imaging, it is limited by its lack of cellular specificity (i.e., expression is also observed in platelets, endothelial cells, and cancer cells) and inability to discriminate between beneficial and toxic immune responses, which must be considered when interpreting results. Therefore, there is a need to discover new functionally relevant imaging biomarkers to specifically quantify different aspects of neuroinflammation.

\section{ALTERNATIVE EXISTING AND EMERGING INNATE IMMUNE-CELL-IMAGING BIOMARKERS}

To overcome the limitations of TSPO PET, other neuroimmune imaging biomarkers are being explored (Fig. 2). Though not described here, MAOB, GSK3, RAGE, MERTK, and S1P1 are also being investigated as PET biomarkers and were recently reviewed (3).

\section{P2X7R}

$\mathrm{P} 2 \mathrm{X}$ ligand-gated ion channel type 7 (P2X7R) is an ionotropic receptor activated by ATP and implicated in microglial proliferation, activation, and apoptosis (11). Under normal physiologic conditions, P2X7R is inactive and predominately expressed by innate immune cells (e.g., macrophages, monocytes, and microglia). Importantly, P2X7R is also found on astrocytes, oligodendrocytes, Schwann cells, and neurons, albeit to a lesser extent (11). After injury, P2X7R expression increases significantly 


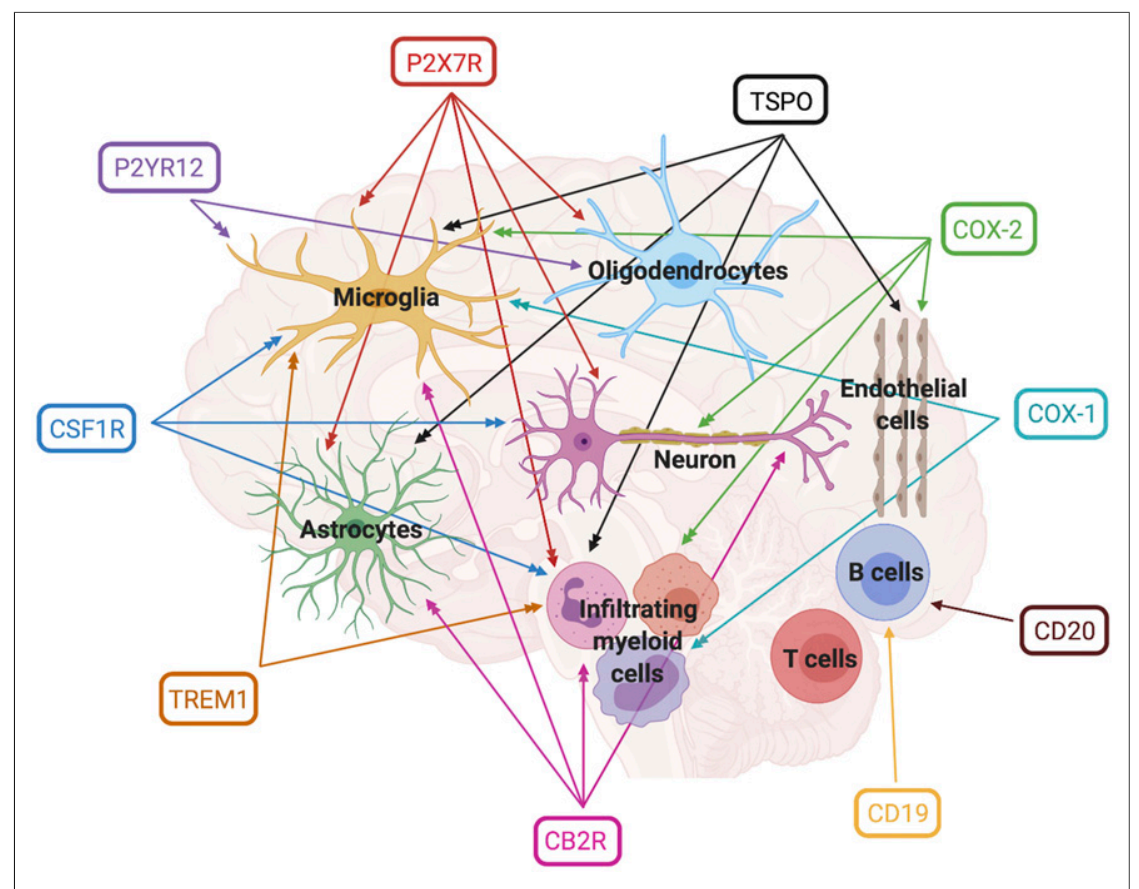

FIGURE 2. Human cellular expression of neuroimmune imaging biomarkers. Single arrowhead indicates basal expression in healthy humans whereas double arrowhead indicates altered expression in the context of neuroinflammation.

on innate immune cells and astrocytes to promote inflammasome formation and release of proinflammatory cytokines and chemokines $(3,11)$. This upregulation and activation of P2X7R are associated with numerous neurodegenerative and neuroinflammatory conditions, including AD, PD, and MS $(11,12)$.

Development of radiotracers for P2X7R is a rapidly expanding area, and there are currently 4 promising radiolabeled $\mathrm{P} 2 \mathrm{X} 7 \mathrm{R}$ antagonists $\left({ }^{11} \mathrm{C}-\mathrm{GSK} 1482160,{ }^{18} \mathrm{~F}-\mathrm{JNJ}-64413739,{ }^{11} \mathrm{C}-\mathrm{JNJ}-\right.$ 54173717, and $\left.{ }^{11} \mathrm{C}-\mathrm{SMW} 139\right)$ that have been validated in rodents and assessed in humans. First-in-human radiation dosimetry studies for ${ }^{11} \mathrm{C}-\mathrm{GSK} 1482160$ demonstrated low uptake in the CNS ( $2 \%$ injected dose/g) and moderate binding in peripheral organs of healthy controls (13). ${ }^{18} \mathrm{~F}-\mathrm{JNJ}-64413739$, on the other hand, showed significant brain uptake with favorable peripheral radiation dosimetry in healthy humans (12). However, high intersubject variability for this tracer and the lack of an identifiable brain reference region may render image quantification challenging. ${ }^{11} \mathrm{C}-\mathrm{JNJ}-54173717$ and ${ }^{11} \mathrm{C}-\mathrm{SMW} 139$ have both been used to image neuroimmune responses in patients. Importantly, ${ }^{11} \mathrm{C}-\mathrm{JNJ}-54173717$ did not detect significant differences between PD or amyotrophic lateral sclerosis (ALS) patients and age-matched healthy controls $(14,15)$. This is possibly due to the suboptimal dynamic range of $\mathrm{P} 2 \mathrm{X} 7 \mathrm{R}$ expression in specific diseases and a reported single-nucleotide polymorphism, rs3751143, which appeared to impact tracer binding in a subset of scanned PD subjects. On the other hand, first-in-human pharmacokinetic studies using ${ }^{11} \mathrm{C}-\mathrm{SMW} 139$ demonstrated increased in vivo binding potential in patients with relapsing remitting MS compared with age-matched healthy controls, warranting further investigation of this tracer (16).

Although P2X7R has a distinct role in inflammasome formation and has been implicated in numerous inflammatory pathways in neurodegenerative diseases, certain factors may limit its overall utility as an imaging biomarker. Similar to TSPO, P2X7R lacks cellular specificity and has moderate basal expression in the healthy CNS, thereby restricting SNR. Moreover, the rs3751143 polymorphism seems to affect the binding affinity of certain P2X7R PET tracers (14). Thus, further clinical investigation is needed in addition to detailed histologic analysis of human brain tissue to better characterize P2X7R expression dynamics in human disease.

\section{CYCLOOXYGENASE}

Cyclooxygenase isoenzymes, cyclooxygenase- 1 and cyclooxygenase-2, are targets of nonsteroidal antiinflammatory drugs and are responsible for catalyzing the conversion of arachidonic acid into prostaglandins. These targets are moderately expressed throughout the healthy CNS $(17,18)$. Recent studies have identified elevated levels of cyclooxygenase-1-positive microglia within (and surrounding) sites of neurodegeneration $(3,19)$. Cyclooxygenase-2 expression is localized to neurons, microglia, and endothelial cells and primarily upregulated in neurons in response to inflammatory stimuli (3).

Multiple cyclooxygenase- 1 inhibitors and their analogs have been radiolabeled for PET imaging. One such analog, ${ }^{11} \mathrm{C}$-ketoprofen-methyl ester, displayed promising cyclooxygenase- 1 selectivity and brain uptake in rats with systemic inflammation (19). Studies on healthy humans demonstrated brain uptake (average $S U V_{\text {peak }}$ of 1.5 after $2 \mathrm{~min}$ ) of ${ }^{11} \mathrm{C}$-ketoprofen-methyl ester, as well as favorable stability and dosimetry for clinical translation (3). However, when assessed in AD patients, no differences in brain uptake or washout were observed, indicating possible low binding affinity or insufficient sensitivity of ${ }^{11} \mathrm{C}$-ketoprofen-methyl ester for detecting cyclooxygenase-1 alterations in vivo (19). More recently, the cyclooxygenase-1-specific radioligand ( ${ }^{11} \mathrm{C}-1,5-b i s-(4-m e t h o x y p h e n y l)-3-(2,2,2-$ tri-fluoroethoxy)$1 \mathrm{H}$-1,2,4-triazole ( ${ }^{11} \mathrm{C}$-PS13) was assessed in rhesus monkeys and demonstrated marked uptake in regions containing high cyclooxygenase- 1 expression, which was significantly blocked by cyclooxygenase-1-selective inhibitors, thereby warranting further evaluation (20).

Several selective cyclooxygenase 2 inhibitors, including rofecoxib and celecoxib, have been radiolabeled and assessed in animal models of neuroinflammation. Most have been unsuccessful because of low brain uptake, nonspecific binding, low affinity, or metabolic instability. However, a recent study reported significant brain uptake of ${ }^{11} \mathrm{C}$-celecoxib in healthy baboons, indicating suitable $\mathrm{BBB}$ penetration (18). In parallel, ${ }^{11} \mathrm{C}-3$-(4-methylsulfonylphenyl)-4phenyl-5-trifluoromethylisoxazole $\left({ }^{11} \mathrm{C}-\mathrm{TMI}\right)$, another radiolabeled cyclooxygenase- 2 inhibitor, was evaluated in healthy rhesus monkeys and also demonstrated sufficient brain uptake and high metabolic stability (17). These tracers have yet to be explored in humans, although preclinical toxicity and dosimetry data indicate that ${ }^{11} \mathrm{C}$-celecoxib is safe for exploratory human studies.

Further characterization of cyclooxygenase-targeted radiotracers in animal models of neuroinflammatory disease and patients is required not only to assess whether they can reliably track pathologic alterations of individual isoforms in vivo but also to ascertain the clinical or diagnostic benefit of doing so. 
$\mathrm{CB} 2 \mathrm{R}$ is moderately expressed on the surface of immune cells in the periphery and at low levels in the healthy CNS, predominantly on glia and endothelial cells, as well as certain subsets of neurons (21). After CNS insult, CB2R expression is markedly upregulated on activated microglia, astrocytes, and neurons (21-23). CB2R upregulation has been implicated in multiple neurologic disorders, and selective activation can attenuate inflammatory responses by reducing proinflammatory cytokine release (21).

Many CB2R radiotracers have been developed and are reviewed in detail by Spinelli et al. (21). One such radiotracer, ${ }^{11} \mathrm{C}-\mathrm{NE} 40$ (2-oxo-7-[ $\left.{ }^{11} \mathrm{C}\right]$ methoxy-8butyloxy-1,2-dihydroquinoline-3-carboxylic acid cyclohexylamide), has been characterized extensively in healthy mice, rats, and rhesus monkeys, whereby it was shown to readily cross the $\mathrm{BBB}$ and bind to $\mathrm{CB} 2 \mathrm{R}$ with high affinity and specificity. Recently, Yamagishi et al. demonstrated that ${ }^{11} \mathrm{C}-\mathrm{NE} 40$ can track neuroinflammation in a mouse model of accelerated aging more accurately than TSPO PET (22). When assessed in healthy humans, ${ }^{11} \mathrm{C}-\mathrm{NE} 40$ exhibited promising brain uptake and washout; however, when used in $\mathrm{AD}$ patients, ${ }^{11} \mathrm{C}-\mathrm{NE} 40$ uptake was not associated with amyloid pathology and, in fact, was reduced in patients compared with controls (24). This finding suggests that ${ }^{11} \mathrm{C}-\mathrm{NE} 40$ may have low in vivo selectivity for $\mathrm{CB} 2 \mathrm{R}$ in humans; alternatively, this finding could reflect a more complex expression profile of $\mathrm{CB} 2 \mathrm{R}$ in clinical AD. Another CB2R tracer, ${ }^{11} \mathrm{C}-\mathrm{A}-836339$ ((2,2,3,3-tetramethylcyclopropanecarboxylic acid [3-(2-[ $\left.{ }^{11} \mathrm{CH}_{3}\right]$ methyloxyethyl)-4, 5-dimethyl-3H-thiazol-(2Z)-ylidene]amide)), developed with higher affinity than ${ }^{11} \mathrm{C}-\mathrm{NE} 40$, has been evaluated in rodents. Increased ${ }^{11} \mathrm{C}-\mathrm{A}-836339$ binding was observed in the brains of transgenic $\mathrm{APP}_{\text {swe }} \times \mathrm{PS}_{\mathrm{dE9}} \mathrm{AD}$ mice compared with wild-type controls (23). In contrast, no significant differences in ${ }^{11} \mathrm{C}-\mathrm{A}-836339$ brain uptake were reported in an ischemic rat model of stroke (21). Clinical assessment of ${ }^{11} \mathrm{C}-\mathrm{A}-836339$ has yet to be reported. Further evaluation is therefore necessary to assess the selectivity and kinetics of the most promising CB2R tracers and determine whether CB2R can be used as a biomarker to sensitively and reliably track neuroinflammation in human CNS diseases.

\section{CSF1R}

Colony-stimulating factor 1 receptor (CSF1R) is a cell surface tyrosine kinase receptor essential for myeloid cell survival, proliferation, and differentiation (25). High basal CSF1R expression in the healthy brain is restricted primarily to microglia and, to a lesser extent, neurons (with conflicting reports on neuronal expression) $(25,26)$. CSF1R levels are significantly increased in diseases associated with marked neuroinflammation, such as $\mathrm{AD}$, MS, and glioblastomas (26).

Initial efforts in CSF1R-specific radiotracer development described the synthesis of ${ }^{11} \mathrm{C}-\mathrm{AZ} 683$, which was hindered by the lack of BBB penetration in vivo (27). More recent work from Horti et al. led to synthesis of the high-affinity CSF1R radioligand 5-cyano- $N$-(4-(4- ${ }^{11} \mathrm{C}$-methylpiperazin-1-yl)-2-(piperidin-1-yl)phenyl)furan-2-carboxamide $\left({ }^{11} \mathrm{C}-\mathrm{CPPC}\right)$. In vivo evaluation of ${ }^{11} \mathrm{C}$ $\mathrm{CPPC}$ in rodent (lipopolysaccharide and experimental autoimmune encephalomyelitis [EAE]) and nonhuman primate (lipopolysaccharide) models of inflammatory disease, as well as in vitro autoradiography of human AD brain tissue (Fig. 3) demonstrated significantly greater signal in the CNS of disease groups than in controls. Although early results appear promising, significant nonspecific binding (i.e., marked PET signal in the brains of CSF1R knockout and

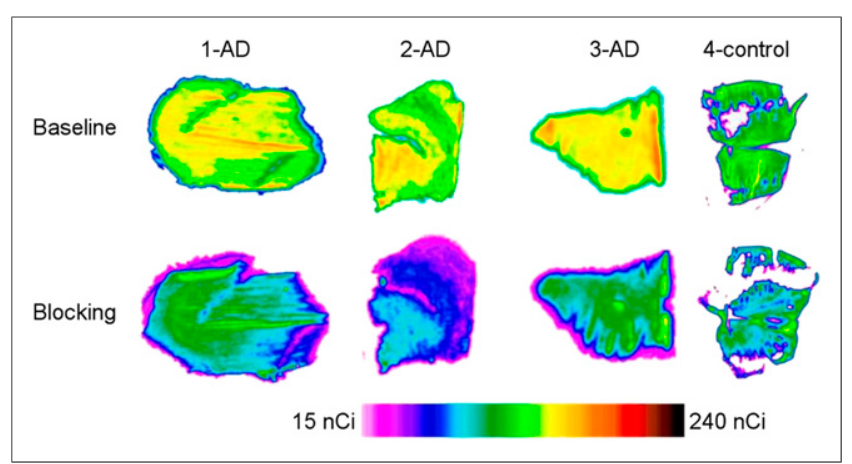

FIGURE 3. In vitro autoradiography examining postmortem human $A D$ brain tissue vs. control using ${ }^{11} \mathrm{C}-\mathrm{CPPC} .1 \mathrm{Ci}=37 \mathrm{GBq}$. (Modified from (25).)

microglia-depleted mice) must be addressed-perhaps by exploring other analogs for future tracer development (25). Moreover, the moderately high basal expression of CSF1R may limit accurate detection of subtle changes in microglial activation in vivo.

\section{P2Y12R}

Purinergic metabotropic 12 receptor (P2Y12R) is a chemoreceptor for adenosine diphosphate expressed peripherally on platelets but localized primarily to microglia within the CNS $(28,29)$. Because of its demonstrated role in microglial chemotaxis and in cytokine and chemokine signaling, in addition to its absence on peripheral immune cells, P2Y12R is an attractive imaging target for visualizing the functional contribution of microglia in different neurologic diseases in vivo. Histologic data show that the specific role and expression patterns of P2Y12R in the CNS are dynamic and nuanced, depending on both disease type and stage. That is, P2Y12R expression can quickly change as microglia shift to adopt different activated phenotypes. For example, P2Y12R expression has been shown to decrease in human postmortem $\mathrm{AD}$-plaque-associated brain tissue (30). However, regional changes in AD tissue appear to be quite dynamic and complex, with a recent study demonstrating that P2Y12R expression decreases in and around dense, mature plaques but increases in the midst of diffuse plaques (29).

To date, only 1 P2YR12 PET radioligand has been developed, ethyl6-(3-(3-((5-chlorothiophen-2-yl)sulfonyl $){ }^{11} \mathrm{C}$-ureido)azetidin-1yl)-5-cyano-2-methylnicotinate, which is an isotopolog of a known high-affinity P2Y12R antagonist. Although autoradiography studies demonstrated the utility of this tracer as a tool for studying P2Y12R in vitro, subsequent in vivo imaging studies in rats revealed low metabolic stability and lack of sufficient brain uptake and retention, possibly due to PGP efflux (31). Of note, ex vivo biodistribution studies of this tracer in mice demonstrated low binding in blood, addressing concerns about the possibility that high uptake in circulating platelets will mask signal within the brain parenchyma. Although further work must be performed to better understand the relationship between P2Y12R expression patterns in different CNS disease states, the high specificity of P2Y12R for microglia in the CNS, in addition to its dynamic functional role, warrants investigation into CNS-penetrant P2Y12R PET radioligands.

\section{TREM1}

Triggering receptor expressed on myeloid cells 1 (TREM1) is a transmembrane immunoglobulin receptor specifically localized to myeloid lineage cells. Interestingly, this receptor appears to be 
absent from microglia in mice (32), though it is present at relatively low levels in healthy human microglia. TREM1 amplifies proinflammatory responses and has shown therapeutic potential in preclinical models of stroke and MS $(32,33)$. Although TREM1 expression is negligible in the healthy murine CNS, it is significantly upregulated on CNS-infiltrating peripheral myeloid cells during neuroinflammation (32). Thus, TREM1 PET could be useful for visualizing peripheral myeloid-cell-driven immune responses in CNS disease.

Our group developed the first TREM1-targeted PET probe $\left({ }^{64} \mathrm{Cu}-\right.$ TREM1-mAb) by radiolabeling a selective anti-TREM1 mAb. After validating its specificity, we demonstrated the ability of ${ }^{64} \mathrm{Cu}$-TREM1mAb to successfully detect CNS-infiltrating peripheral myeloid cells before symptom manifestation in EAE mice with greater sensitivity than TSPO PET (32). Similarly, we showed that in a mouse model of stroke, TREM1 PET detected increased signal in ischemic brain tissue, spleen, and intestines of stroked mice (33). These results led to further investigation of TREM1 expression on intestinal macrophages, where it was shown to play a role in gut permeability and bacterial translocation after stroke. This work highlights the utility of TREM1 PET for characterizing systemic innate immune responses in the context of neurologic disease. Clinical translation of ${ }^{64} \mathrm{Cu}$-TREM1-mAb and development of small-molecule candidates are warranted for imaging TREM1 in diseases with and without CNS-infiltrating myeloid cells.

\section{ADAPTIVE IMMUNE-CELL-IMAGING BIOMARKERS}

Adaptive immune cells (e.g., B and T lymphocytes) play a critical role in several neurologic disorders. In recent years, B-cell-targeted therapies have shown remarkable results in improving clinical symptoms of MS (34). Research has shown that CNS-infiltrating $\mathrm{B}$ cells are key drivers of maladaptive immune responses in MS by increasing antigen presentation and stimulating autoimmune $\mathrm{T}$ cells (34). Considering the promise of anti-B-cell therapies, cell-specific in vivo imaging of $\mathrm{B}$ cells could be an essential tool for patient stratification and MS therapy monitoring.

Our group has pioneered methods to image the adaptive immune response in the CNS by radiolabeling antibodies specific for 2 B-cell antigens, both of which have been targeted successfully in clinical trials for MS therapy: cluster differentiation factor 20 (CD20) and cluster differentiation factor 19 (CD19). CD20 and CD19 are transmembrane proteins involved in B-cell immune responses. CD20 is not expressed on B cells during their earliest and latest stages of development, whereas CD19 is expressed across development on pre-B cells, plasmablasts, and a subset of long-lived plasma cells $(34,35)$. We developed a $\mathrm{CD} 20$ PET radiotracer, ${ }^{64} \mathrm{Cu}$-rituximab, which was administered to transgenic mice expressing human CD20 induced with EAE to mimic MS autoimmune disease. In vivo imaging and ex vivo studies confirmed increased binding of this tracer in spinal cords and brains of EAE mice compared with naïve controls (36). More recently, we also generated and assessed the first CD19 PET radiotracer, ${ }^{64} \mathrm{Cu}-\mathrm{CD} 19-$ $\mathrm{mAB}$. Imaging in EAE mice demonstrated significant binding in the CNS of diseased mice compared with naive controls, correlating histologically with numbers of B cells (35). These studies lay the groundwork for future in vivo clinical investigations of imaging B cells in the context of CNS pathology.

\section{CONCLUSIONS AND FUTURE DIRECTIONS}

Considering the growing emphasis on developing immunomodulatory therapies for neurologic diseases, there is a clear need for noninvasive imaging tools that can monitor responses to such therapies and stratify eligible patients for clinical trials. Despite its limitations, TSPO PET remains the most widely used tool for assessing neuroinflammation in clinical studies and has provided valuable insight into the pathophysiology of immune responses in relation to cognitive decline across many neurologic disorders. Informed by the shortcomings of TSPO PET, new biomarkers should be identified and validated according to the characteristics needed to answer critical biologic and clinical questions of interest. For example, questions such as, "Are activated microglia driving cognitive decline in AD?" or, "What is the relative contribution of microglia versus peripheral myeloid cells to MS pathology?" require highly specific biomarkers that delineate between CNS resident microglia and peripheral CNS-infiltrating myeloid cells. TSPO does not fulfill this requirement. Thus, it remains necessary to identify biomarkers that have high cell-type specificity and reflect specific immune-cell functional phenotypes. This is in addition to having an adequate dynamic range to enable detection of such phenomena in vivo. A few of the emerging PET imaging biomarkers mentioned in this review, including CSF1R and TREM1, have the potential to overcome some of the limitations of TSPO PET and provide more precise measures of specific immune cell function; however, further validation is still necessary.

In addition to the biomarkers discussed, other innate immune targets are also being explored, including CD11b, found exclusively on activated myeloid lineage leukocytes. To date, this target has been explored primarily in the periphery, but a study recently demonstrated favorable brain uptake of the ${ }^{89} \mathrm{Zr}$-anti-CD11b antibody radiotracer in a murine glioblastoma model (37). Additionally, considering the known infiltration and neurotoxic capabilities of adaptive immune lymphocytes in both autoimmune and neurodegenerative disorders, further work must consider development and evaluation of additional adaptive immune imaging agents for CNS applications. T-cell imaging, for example, has primarily been used in cancer detection and immunotherapy monitoring, via targeting CD4, CD8, and OX40 (38,39).

Here, we have summarized the potential of various neuroinflammation imaging biomarkers and emphasized a growing need to extensively validate them as clinically informative tools before developing radiotracers. Future work will require a more detailed discussion around the nuanced microglial phenotypes when evaluating neuroimmune imaging biomarkers. Although previously assigned to the binary pro- or antiinflammatory axis, it is now clear that microglia are significantly more complex than the prescribed M1/M2 paradigm, where proinflammatory or antiinflammatory can refer to a continuum of activation states with both maladaptive and beneficial phenotypes. Thus, there is a call to action for the field of neuroimmune PET imaging to identify and validate biomarkers that better capture these complex and dynamic immune cell activation states in order to more accurately investigate disease progression and treatment response $(1,2)$. In addition, tracer design and development using computational BBB permeability modeling tools (such as the multiparameter optimization or artificial intelligence algorithms) in conjunction with high-throughput screening will significantly improve the success rate of CNS PET imaging agents. Cutting-edge technologies such as human BBB microfluidic devices and BBB organoids could also provide critical cost-effective tools to systematically evaluate tracer BBB permeability in vitro. Furthermore, in vitro target validation using postmortem human tissue remains critically important (although tissue can be limited or difficult to obtain for 
certain diseases) to better understand the utility of new biomarkers and radiotracers in disease before in vivo clinical evaluation.

In parallel, the development of new clinical imaging systems, including time-of-flight PET/MRI and the groundbreaking total-body PET, will substantially improve image resolution and sensitivity, thus enabling enhanced detection of targets with low endogenous expression, subtle alterations, or diffuse expression in the CNS. This innovation will also increase the translation and accessibility of CNS PET imaging by reducing the required radioactive dose by orders of magnitude, shortening scan times, and eliminating the need for invasive arterial plasma sampling for input function determination. Moreover, total body imaging will enable simultaneous monitoring of peripheral and central immune responses in order to understand the relative contribution and interplay of each in CNS pathology (40).

\section{DISCLOSURE}

Financial support was received from NIH/NINDS (R21NS109783, to Michelle James). No other potential conflict of interest relevant to this article was reported.

\section{REFERENCES}

1. Werry EL, Bright FM, Piguet O, et al. Recent developments in TSPO PET imaging as a biomarker of neuroinflammation in neurodegenerative disorders. Int J Mol Sci. 2019;20:3161.

2. Heppner FL, Ransohoff RM, Becher B. Immune attack: the role of inflammation in Alzheimer disease. Nat Rev Neurosci. 2015;16:358-372.

3. Narayanaswami V, Dahl K, Bernard-Gauthier V, Josephson L, Cumming P, Vasdev N. Emerging PET radiotracers and targets for imaging of neuroinflammation in neurodegenerative diseases: outlook beyond TSPO. Mol Imaging. 2018; 17:1536012118792317.

4. Wager TT, Hou X, Verhoest PR, Villalobos A. Moving beyond rules: the development of a central nervous system multiparameter optimization (CNS MPO) approach to enable alignment of druglike properties. ACS Chem Neurosci. 2010;1: 435-449.

5. Kreisl WC, Henter ID, Innis RB. Imaging translocator protein as a biomarker of neuroinflammation in dementia. Adv Pharmacol. 2018;82:163-185.

6. Yokokura M, Terada T, Bunai T, et al. Depiction of microglial activation in aging and dementia: positron emission tomography with ${ }^{11} \mathrm{C}$-DPA713 versus ${ }^{11} \mathrm{C}-(\mathrm{R})$ PK11195. J Cereb Blood Flow Metab. 2017;37:877-889.

7. Chaney A, Cropper HC, Johnson EM, et al. ${ }^{11}$ C-DPA-713 versus ${ }^{18}$ F-GE-180: a preclinical comparison of translocator protein $18 \mathrm{kDa}$ PET tracers to visualize acute and chronic neuroinflammation in a mouse model of ischemic stroke. $J$ Nucl Med. 2019;60:122-128.

8. Fujita M, Kobayashi M, Ikawa M, et al. Comparison of four ${ }^{11} \mathrm{C}$-labeled PET ligands to quantify translocator protein $18 \mathrm{kDa}$ (TSPO) in human brain: $(R)$-PK11195, PBR28, DPA-713, and ER176 - based on recent publications that measured specific-to-non-displaceable ratios. EJNMMI Res. 2017;7:84.

9. Loggia ML, Chonde DB, Akeju O, et al. Evidence for brain glial activation in chronic pain patients. Brain. 2015;138:604-615.

10. Jucaite A, Svenningsson P, Rinne JO, et al. Effect of the myeloperoxidase inhibitor AZD3241 on microglia: a PET study in Parkinson's disease. Brain. 2015;138: 2687-2700.

11. Tewari M, Seth P. Emerging role of P2X7 receptors in CNS health and disease. Ageing Res Rev. 2015;24:328-342.

12. Koole M, Schmidt ME, Hijzen A, et al. ${ }^{18}$ F-JNJ-64413739, a novel PET ligand for the P2X7 ion channel: radiation dosimetry, kinetic modeling, test-retest variability, and occupancy of the P2X7 antagonist JNJ-54175446. J Nucl Med. 2019;60:683-690.

13. Green M, Hutchins G, Fletcher J, et al. Distribution of the P2X7-receptortargeted ${ }^{11} \mathrm{C}$-GSK1482160 radiopharmaceutical in normal human subjects [abstract]. J Nucl Med. 2018;59(suppl 1):1009.

14. Van Weehaeghe D, Koole M, Schmidt ME, et al. ${ }^{11}$ C-JNJ54173717, a novel $\mathrm{P} 2 \mathrm{X} 7$ receptor radioligand as marker for neuroinflammation: human biodistribution, dosimetry, brain kinetic modelling and quantification of brain $\mathrm{P} 2 \mathrm{X} 7$ receptors in patients with Parkinson's disease and healthy volunteers. Eur J Nucl Med Mol Imaging. 2019;46:2051-2064.

15. Van Weehaeghe D, Van Schoor E, De Vocht J, et al. TSPO versus P2X7 as target for neuroinflammation: an in vitro and in vivo study. J Nucl Med. 2020;61:604-607.
16. Hagens MHJ, Golla SSV, Janssen $\mathrm{B}$, et al. The $\mathrm{P} 2 \mathrm{X}_{7}$ receptor tracer ${ }^{11} \mathrm{C}$ SMW139 as an in vivo marker of neuroinflammation in multiple sclerosis: a first-in man study. Eur J Nucl Med Mol Imaging. 2020;47:379-389.

17. Kumar JSD, Zanderigo F, Prabhakaran J, Rubin-Falcone H, Parsey RV, Mann JJ. In vivo evaluation of ${ }^{11} \mathrm{C}$-TMI, a COX-2 selective PET tracer, in baboons. Bioorg Med Chem Lett. 2018;28:3592-3595.

18. Kumar JSD, Bai B, Zanderigo F, et al. In vivo brain imaging, biodistribution, and radiation dosimetry estimation of ${ }^{11} \mathrm{C}$-celecoxib, a COX-2 PET ligand, in nonhuman primates. Molecules. 2018;23:1929.

19. Ohnishi A, Senda M, Yamane T, et al. Exploratory human PET study of the effectiveness of ${ }^{11} \mathrm{C}$-ketoprofen methyl ester, a potential biomarker of neuroinflammatory processes in Alzheimer's disease. Nucl Med Biol. 2016;43:438-444.

20. Kim MJ, Shrestha SS, Cortes M, et al. Evaluation of two potent and selective PET radioligands to image COX-1 and COX-2 in rhesus monkeys. $\mathrm{J} \mathrm{Nucl} \mathrm{Med}$. 2018;59:1907-1912.

21. Spinelli F, Mu L, Ametamey SM. Radioligands for positron emission tomography imaging of cannabinoid type 2 receptor. J Labelled Comp Radiopharm. 2018;61:299-308.

22. Yamagishi S, Iga Y, Nakamura M, et al. Upregulation of cannabinoid receptor type 2, but not TSPO, in senescence-accelerated neuroinflammation in mice: a positron emission tomography study. J Neuroinflammation. 2019;16:208.

23. Savonenko AV, Melnikova T, Wang Y, et al. Cannabinoid CB2 receptors in a mouse model of $\mathrm{A} \beta$ amyloidosis: immunohistochemical analysis and suitability as a PET biomarker of neuroinflammation. PLoS One. 2015;10:e129618.

24. Ahmad R, Postnov A, Bormans G, Versijpt J, Vandenbulcke M, Van Laere K. Decreased in vivo availability of the cannabinoid type 2 receptor in Alzheimer's disease. Eur J Nucl Med Mol Imaging. 2016;43:2219-2227.

25. Horti AG, Naik R, Foss CA, et al. PET imaging of microglia by targeting macrophage colony-stimulating factor 1 receptor (CSF1R). Proc Natl Acad Sci USA. 2019;116:1686-1691.

26. Chitu V, Gokhan S, Nandi S, Mehler MF, Stanley ER. Emerging roles for CSF-1 receptor and its ligands in the nervous system. Trends Neurosci. 2016;39:378-393.

27. Tanzey SS, Shao X, Stauff J, et al. Synthesis and initial in vivo evaluation of ${ }^{11} \mathrm{C}-$ AZ683: a novel PET radiotracer for colony stimulating factor 1 receptor (CSF1R). Pharmaceuticals (Basel). 2018;11:136.

28. Beaino W, Janssen B, Kooij G, et al. Purinergic receptors P2Y12R and P2X7R: potential targets for PET imaging of microglia phenotypes in multiple sclerosis. J Neuroinflammation. 2017;14:259.

29. Walker DG, Tang TM, Mendsaikhan A, et al. Patterns of expression of purinergic receptor P2RY12, a putative marker for non-activated microglia, in aged and Alzheimer's disease brains. Int J Mol Sci. 2020;21:678.

30. Mildner A, Huang H, Radke J, Stenzel W, Priller J. P2 $\mathrm{Y}_{12}$ receptor is expressed on human microglia under physiological conditions throughout development and is sensitive to neuroinflammatory diseases. Glia. 2017;65:375-387.

31. Villa A, Klein B, Janssen B, et al. Identification of new molecular targets for PET imaging of the microglial anti-inflammatory activation state. Theranostics. 2018; 8:5400-5418.

32. Chaney AM, Cropper HC, Johnson EM, et al. Tracking the invaders in multiple sclerosis: a new highly specific PET imaging approach for visualizing peripheral innate immune activation with higher sensitivity than TSPO-PET. Presented at: World Molecular Imaging Congress 2019; September 4-7, 2019; Montreal, Quebec, Canada.

33. Liu Q, Johnson EM, Lam RK, et al. Peripheral TREM1 responses to brain and intestinal immunogens amplify stroke severity. Nat Immunol. 2019;20:10231034 .

34. Milo R. Therapies for multiple sclerosis targeting B cells. Croat Med J. 2019;60: 87-98.

35. Stevens MY, Cropper HC, Jackson IM, et al. Radiolabeling and pre-clinical evaluation of a first-in-class CD19 PET tracer for imaging B cells in multiple sclerosis [abstract]. J Nucl Med. 2019;60(suppl 1):129.

36. James ML, Hoehne A, Mayer AT, et al. Imaging B cells in a mouse model of multiple sclerosis using ${ }^{64} \mathrm{Cu}$-rituximab PET. J Nucl Med. 2017;58:1845-1851.

37. Nigam S, McCarl L, Kumar R, et al. Preclinical immunoPET imaging of glioblastoma-infiltrating myeloid cells using zirconium-89 labeled anti-CD11b antibody. Mol Imaging Biol. 2020;22:685-694.

38. Alam IS, Mayer AT, Sagiv-Barfi I, et al. Imaging activated T cells predicts response to cancer vaccines. J Clin Invest. 2018;128:2569-2580.

39. Kristensen LK, Fröhlich C, Christensen C, et al. CD4 ${ }^{+}$and CD8a ${ }^{+}$PET imaging predicts response to novel PD-1 checkpoint inhibitor: studies of Sym021 in syngeneic mouse cancer models. Theranostics. 2019;9:8221-8238.

40. Cherry SR, Jones T, Karp JS, Qi J, Moses WW, Badawi RD. Total-body PET: maximizing sensitivity to create new opportunities for clinical research and patient care. J Nucl Med. 2018;59:3-12. 